\title{
Extraction of Road Using Morphological Enhancement
}

\author{
${ }^{1}$ Harisha K S, \\ Assistant Professor, \\ Dept. of E\&CE, \\ Government Engineering College, \\ Haveri, Karnataka, India
}

\begin{abstract}
Extraction of Roads, Rivers and other map objects is an important step in many military and civilian applications. In this process the information is extracted which possess high efficiency and accuracy and is fed into GIS (Geographical Information System). In this paper, we have explored different algorithms with better efficiency and accuracy. Road extraction can take place for two kinds of roads namely urban and non-urban roads. Urban roads are more complex to analyze because of their architectural complexity, occlusions created by trees, heavy traffic and extensive network, whereas non-urban roads are easier to analyze because of less structural complexity. The proposed algorithm exploits the properties of road segments to develop customized operators to accurately derive the road segments. The customized operators include directional morphological enhancement, directional segmentation and thinning. The proposed algorithm is systematically evaluated on the basis of variety of images and compared with other algorithms (Canny, Sobel, Roberts, and Morphological Segmentation). The results demonstrate that the algorithm proposed is both accurate and efficient.
\end{abstract}

Keywords: Road extraction; High resolution satellite image; Connected component; Morphological operation; Trivial opening; Completeness; Correctness; Quality.

\section{Introduction}

Since the first American land observation satellite launched in 1982, all kinds of technologies applied to the RS image processing have developed rapidly, including image compression, transmission, classification, fusion and understanding. All of those high resolution RS images such as I Konos, Quick Bird, World View and Geo Eye create a quick and economical way to access the newly acquired geographic information, and lay a very important basis for the further applications of RS technology. The applications of the high resolution RS image processing mainly include the following aspects: city remote sensing, basic geographic mapping, environmental monitoring and assessment, precision agriculture, and public information service, etc. The goal of the RS applications is to extract information and identify interested targets to complete image understanding. The road extraction from a RS image is a challenging but important research topic.. The research of road extraction is of great significance for traffic management, city planning, road monitoring, GPS navigation and map updating, etc. This paper makes a summary of different road extraction. 


\section{Problem statement}

Urban roads are more complex to analyze because of their architectural complexity occlusions created by trees, heavy traffic and extensive network, whereas non-urban roads are easier to analyze because of less structural complexity. The history of road extraction can be linked to the earlier year of 70's. When remote sensing and its applications came into use the need of extraction was felt, since the researchers started working in the area of image processing. Firstly, road extraction was done manually wherein a person was appointed to highlight and draw roads and its features manually but as the process was very cumbersome the need for automation was felt hence came semi-automatic and automatic techniques. In semi -automatic technique image processing, segmentation, classification, morphological segmentation came into light. After the era of semiautomatic road extraction came the era of automatic road detection techniques which involved learning algorithms and artificial intelligence. In automated road detection neural networks were used to give effective and efficient results with least human interference. These developments in the field of road extraction have increased the efficiency and accuracy of the targeted output but because of its vast application there can still be huge development and improvement. The main disadvantage of different given methods is the difficulty to provide the best parameters for a particular given image.

\section{Literature Survey}

HU Hua, LIU Ying., (2008), improved the traditional algorithm and presented a new method which uses a multi-weighted terms to judge the road edges s, makes full use of the

characters of triangle-units. Then, the triangle-units belonging to road section were extracted dependingon the feature parameters. Finally, road edges and road centerlines were automatically extracted by road triangle-units. physical characteristics of the road, and makes the context of road edge pixels as a judgment, in order to recognize the edge segments of the road. Bridge- link mode accord to the characteristics of the road: two parallel lines and certain width. To connect the two marginalized segment of the corresponding pixels in order to achieve road extraction .Experimental results demonstrate the algorithm can eliminate noise, effectively improve the accuracy and velocity of the road extraction.

Jose Hormese and Dr C.Saravanan (2016), proposed automated road extraction method. In this method, a Vectorization Approach for the automatic method of road extraction is being used where the image is segmented to identify the road network regions followed by a decision making and continuity procedure to correctly detect the roads and the Vectorization step to identify the line segments or curved segments which represents the road. This method may be employed for obtaining information for feeding large-scale Geographic Information System. In the automatic method of road extraction the extracted roads are converted into road vectors in order to use these vector road maps in GIS. A semiautomated scheme is used for scenarios where fully automated system fails. A combination of both methods can be devised for a full-fledged real business scenario.

Wang Zhendong (2018), suggests a new road extraction method from high resolution satellite image based on Delaunay algorithms was proposed in this paper. Firstly, the images should be pre-treated by edge detection and binarization, etc. Secondly, the Delaunay triangulations were constructed according to the Delaunay triangulation algorithm, which could represent the image information. Each triangle in Delaunay triangulation was defined as the basic processing unit, namely triangle unit. Thirdly, combined with the road features, the four feature parameters were put forward after analysing and summarizing the

Zelang et al., (2013), Proposed a road extraction approach which is based on shape features and multivariate adaptive regression splines (MARS). The measurement of optimally oriented flux (OOF) helps to eliminate the undesired spurs for the selection of 
end points from a classified image and further these end points are connected accurately to formulate the road network using a geodesic method.

Singh and Garg,(2013) The controlling parameters have utilized in the fuzziness of the FCM approach, which help to estimate the segmented road results and thereafter Stentiford thinning algorithm (STA) is used to estimate the road network from classified results. Such improvements facilitate FCM method manipulation and lead to segmentation that is more robust.

M.Revathi And M.Sharmila(2013), they proposed a method where there are two approaches for road extraction based on Level Set and Mean Shift methods are proposed. The image is pre-processed to improve the tolerance by reducing the noise (the buildings, parking lots, vegetation regions and other open spaces) and roads are first extracted as elongated regions, non-linear noise segments are removed using a median filter (based on the fact that road networks constitute large number of small linear structures). Then road extraction is performed using Level Set and Mean Shift method. Finally the accuracy for the road extracted images is evaluated based on quality measures. The $1 \mathrm{~m}$ resolution IKONOS data has been used for the experiment.

Guilherme Pina Cardim, Erivaldo Antônio da Silva and Mauricio Araújo Dias (2018), proposed a statistical evaluation and analysis of road extraction methodologies using a common image dataset, to achieve this goal a

dataset containing remote sensing images of three different types of roads i.e., highways, city roads and rural paths, a group of images from the ISPRS (International Society for Photogrammetry and Remote Sensing) dataset. Furthermore, three road extraction methodologies were selected from the literature, in accordance with their availability, to be processed and evaluated using well-known statistical metrics. The achieved results are encouraging and indicate that the proposed statistical evaluation and analysis can allow researchers to evaluate and compare road extraction methodologies using this common dataset extracting similar characteristics to obtain a more reliable comparison among them.
Sghaier and Lepage (2016), they performed a multiscale decomposition using the beam let transformation that allows the identification of the most appropriate scale for each road segment calculation, and the segments position and direction. Summarizing, the beam let transformation consists of successive divisions of the image by four in a recursive way until it obtains blocks of $2 \times 2$ pixels. The former division of the image leads to the requisite of processing images or sub images with a size equal to a number power of two.

\section{Objectives}

The primary objectives of the thesis are:

1. To collect the large amount of dataset for different satellite images of road work.

2. To create the data base of road work for feature extraction.

3. To introduce the efficient algorithm for road work extraction.

4. To apply few image processing techniques (using Matlab) such as line detection techniques like Sobel, Canny and Roberts's edge detection for the improvement of correctness and accuracy of the processed road images.

5. Finally to compare the current implementation with the existing works in terms of accuracy and correctness.

\section{Methodology of the work}

In this Algorithm, first step is the creation of database. The database should contain satellite road images whose road intensity values are within a particular range. By considering different intensity ranges, any type of roads can be extracted. The basic steps involved in the algorithm are described in Figure (1). The basic steps involved are

1. The given input image is converted to grayscale image and then the grayscale image is adjusted to the threshold values of the road.

2. The obtained image is then converted to binary image with threshold value taken from graythresh () i.e., Otsu's method.

3. Then the image is filtered using a median filter to remove noises and it still contains unwanted objects 
and those are removed using morphological operations.

4. The edges of the extracted road are determined and finally the extracted road is overlaid onto the original image.

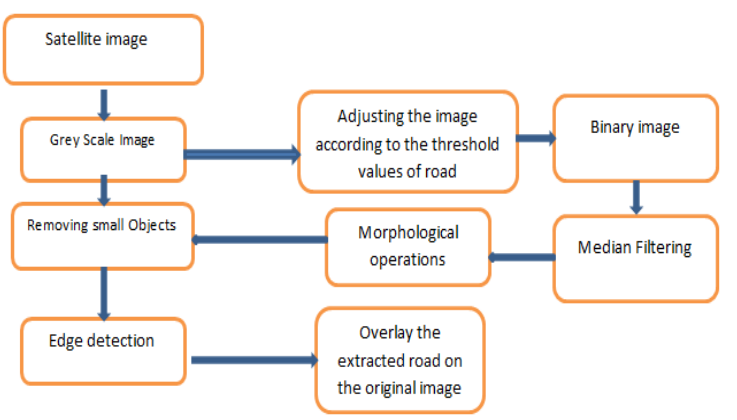

Figure1:Process of satellite image Representation in flow chart

At first the image is converted into grayscale image and then it is adjusted from threshold range 0.5 to 0.9 as most of the roads contain this range to remove unwanted stuff. Figure 4 shows the image obtained after adjusting. Then the image is converted to binary image using 'graythresh' i.e., Otsu's method which automatically sets the threshold value for the conversion. The obtained image after converting to binary. In Otsu's method we exhaustively search for the threshold that minimizes the intra-class variance (the

variance within the class), defined as a weighted sum of variances of the two classes:

Figure1

\section{Results and discussions}

The output images will be displayed in separate figure windows one after the other.

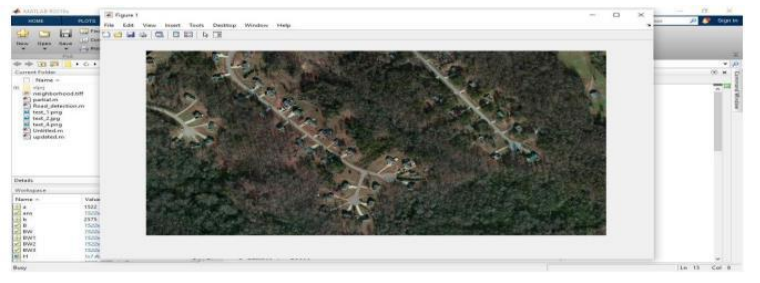

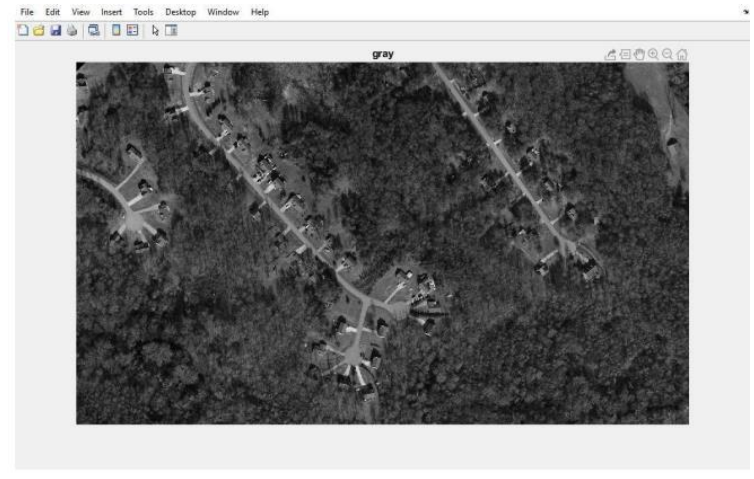

Figure 2: Original input satellite image

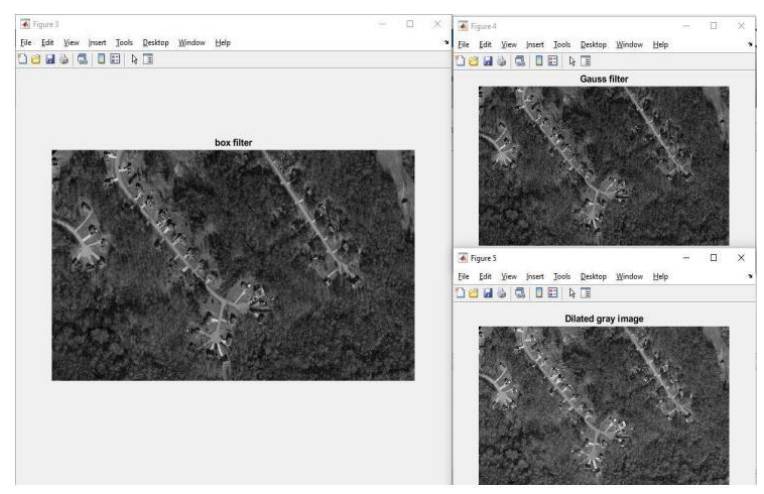

Figure 3: Grayscale Image

Next the Gaussian filtered, Box filtered and dilated image outputs are obtained

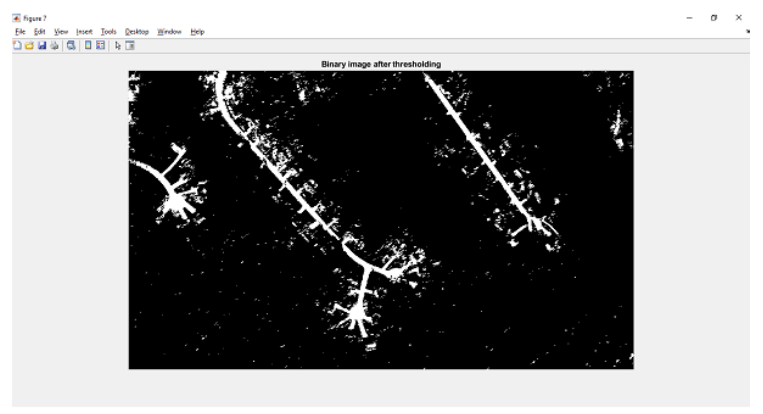

Figure 4: Binary Image after Thresholding

for the above Figure, Median filter is applied and also unwanted noise is removed by filling the white noise present around the roads using hole filling. 


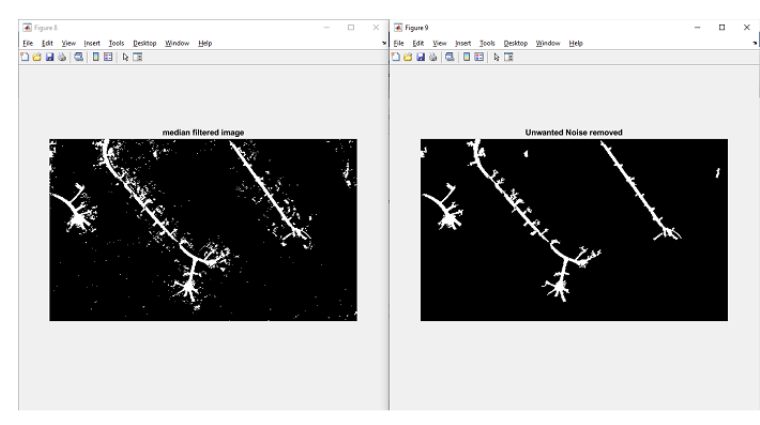

Figure 5: Median filtered and noise removed images

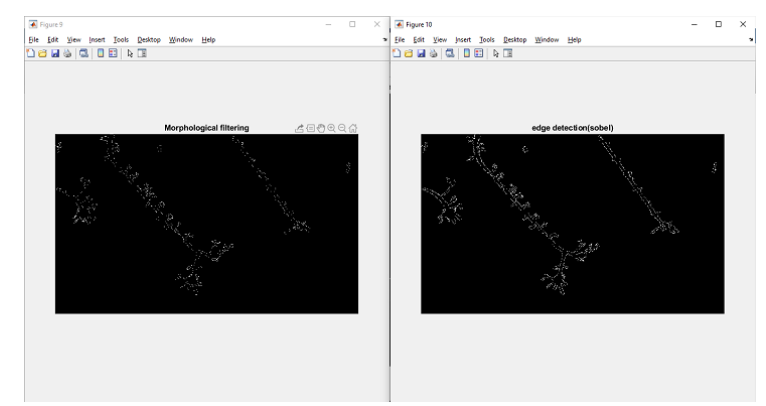

Figure 6: Morphological filtering and Sobel edge detection outputs

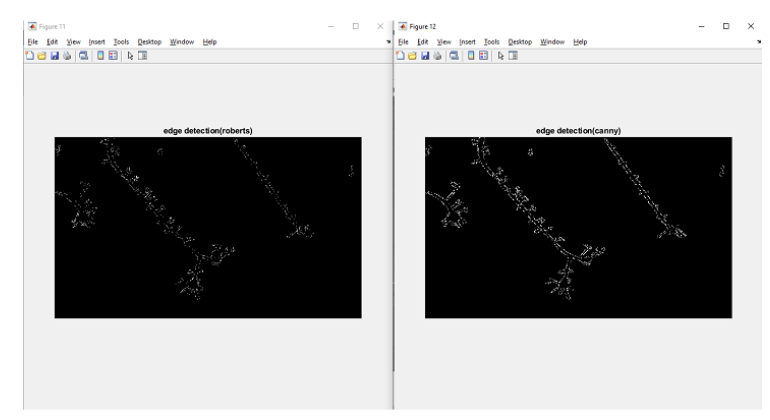

Figure 7: Roberts and Canny edge detection outputs

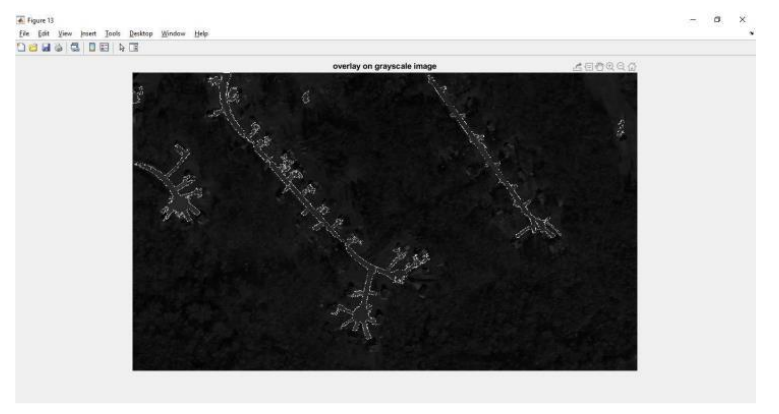

Figure 8: Edges detected overlapped on Gray Scale image
The database for road extraction can be created based on the color of the roads. MATLAB R2019a software platform is used to perform the road extraction. From the final Figure it is clear that some of the objects other than roads are also detected. This is because those objects are also having the color within the particular range as that of roads. These objects could be small parts of barren land and parking lots.

\section{Conclusion}

In this paper, we developed an efficient algorithm to extract the roads from high resolution satellite images. The roads play a vital role in urban planning. The algorithm introduced is automatic one. It requires only very little interaction from the users. The algorithm was implemented to detect roadways from satellite images with resolution greater than $0.5 \mathrm{~m}$. The important and key parameter of this algorithm is the color of the roads in the database. Different types of roads can be extracted based on this algorithm. Since extraction is solely based on color, some of the barren lands and small areas of parking lots are also being extracted. This is because the locations also have the same pixel intensity values as that of roads. Different other techniques such as usage of Digital Elevation Models, active contours and artificial intelligence methods could be included to remove the unwanted objects that are being extracted. The algorithm implemented is fast, robust and easy to understand and implement

\section{References}

1) Cao, C.; Sun, Y. Automatic road centerline extraction from imagery using road GPS data. Remote Sens. 2014,6, 9014-9033.

2) Sghaier, M.O., Lepage R, Road extraction from very high resolution remote sensing optical images based on texture analysis and 
Beamlet transform. IEEE J. Sel. Top. Appl. Earth Obs. Remote Sens. 2016.

3) Kaur, A.; Singh, R. various methods of road extraction from satellite images: A review. Int. J. Res. 2015, 2, 1025-1032.

4) Cheng, G.; Han, J.; Lu, X. Remote sensing image scene classification: Benchmark and state of art. Proc. IEEE 2017, 105, 1865-1883.

5) Ma, H.; Cheng, X.; Wang, X.; Yuan, J. Road information extraction from high resolution remote sensing images based on threshold segmentation and mathematical morphology. In Image and Signal Processing (CISP), Proceedings of 6th International Congress on Image and Signal Processing (CISP 2013), Hangzhou, China, 16-18 December 2013; IEEE: Hangzhou, China, 2013; pp. 626-630.

6) Cardim, G.P.; da Silva, E.A.; Dias, M.A.; Bravo, I. Semiautomatic methodology for cartographic feature extraction using high-resolution remote sensing images. Revista UD y la Geomatica 2014, 8, 53-58.

7) M. Revathi and M. Sharmila, "Automatic road extraction using high resolution satellite images based on level set and mean shift methods," in 2013 Fourth International Conference on Computing, Communications and Networking Technologies (ICCCNT), Tiruchengode, India, 2013 pp. 1-7. doi:10.1109/ICCCNT.2013.6726766.

8) Z. Wang, X. Liu, L. Liu and J. Shi, "A Method of Road Extraction from High Resolution Remote Image Based on Delaunay Algorithms," in 2018 International Conference on Robots \& Intelligent System (ICRIS), Changsha, China, 2018 pp. 127-130. doi: 10.1109/ICRIS.2018.00040 
